*For correspondence: heuer@ genzentrum.Imu.de (AH); ed. hurt@bzh.uni-heidelberg.de (EH); beckmann@genzentrum.Imu.de (RB)

${ }^{\dagger}$ These authors contributed equally to this work

Present address: ${ }^{\ddagger}$ University of Sheffield, Sheffield, United Kingdom

Competing interests: The authors declare that no competing interests exist.

Funding: See page 12

Received: 05 July 2017

Accepted: 03 November 2017

Published: 20 November 2017

Reviewing editor: Rachel Green, Johns Hopkins School of Medicine, United States

(c) Copyright Heuer et al. This article is distributed under the terms of the Creative Commons Attribution License, which permits unrestricted use and redistribution provided that the original author and source are credited.

\section{Cryo-EM structure of a late pre-40S ribosomal subunit from Saccharomyces cerevisiae}

\author{
André Heuer ${ }^{1 \dagger \star}$, Emma Thomson ${ }^{2 \dagger \neq}$, Christian Schmidt ${ }^{1}$, Otto Berninghausen ${ }^{1}$, \\ Thomas Becker ${ }^{1}$, Ed Hurt ${ }^{1,2 \star}$, Roland Beckmann ${ }^{1 *}$
}

${ }^{1}$ Gene Center Munich, Department of Biochemistry, University of Munich, Munich, Germany; ${ }^{2}$ Heidelberg University Biochemistry Center, Heidelberg University, Heidelberg, Germany

\begin{abstract}
Mechanistic understanding of eukaryotic ribosome formation requires a detailed structural knowledge of the numerous assembly intermediates, generated along a complex pathway. Here, we present the structure of a late pre- $40 \mathrm{~S}$ particle at $3.6 \AA$ resolution, revealing in molecular detail how assembly factors regulate the timely folding of pre-18S rRNA. The structure shows that, rather than sterically blocking $40 \mathrm{~S}$ translational active sites, the associated assembly factors Tsr1, Enp1, Rio2 and Pno1 collectively preclude their final maturation, thereby preventing untimely tRNA and mRNA binding and error prone translation. Moreover, the structure explains how Pno1 coordinates the 3'end cleavage of the 18S rRNA by Nob1 and how the late factor's removal in the cytoplasm ensures the structural integrity of the maturing $40 \mathrm{~S}$ subunit.
\end{abstract}

DOI: https://doi.org/10.7554/eLife.30189.001

\section{Introduction}

Ribosomes are the cellular machines that translate mRNAs into proteins. In eukaryotes, they consist of a small $40 \mathrm{~S}$ and large $60 \mathrm{~S}$ subunit, which carry the decoding and peptidyl transferase activity, respectively, and together comprise four ribosomal (r)RNAs (18S, 5.8S, 25S and 5S rRNA) and 78 ribosomal proteins in yeast. The synthesis of eukaryotic ribosomal subunits requires the concerted activity of $\sim 200$ assembly factors that drive ribosome biogenesis through a series of pre-rRNA cleavage, folding and modification reactions, which are coupled to the incorporation of ribosomal proteins (Henras et al., 2015; Woolford and Baserga, 2013; Zemp and Kutay, 2007). Initial steps of $40 \mathrm{~S}$ biogenesis occur in the nucleolus, which leads to the formation of the first stable assembly intermediate, called the $90 \mathrm{~S}$ pre-ribosome (Dragon et al., 2002; Grandi et al., 2002; Kornprobst et al., 2016), within which many of the early assembly steps for the $40 S$ take place. This process requires between 50-70 different ribosome biogenesis factors (RBFs) (Woolford and Baserga, 2013; Grandi et al., 2002), which were shown by recent cryo-electron microscopy (cryo-EM) analysis to engulf the nascent pre-18S rRNA (Kornprobst et al., 2016; Sun et al., 2017; Chaker-Margot et al., 2017). Following early maturation steps, the pre-40S moiety detaches and is subsequently exported to the cytoplasm, containing only a handful of biogenesis factors including Pno1, Tsr1, Enp1, Ltv1, Nob1, Dim1 and Rio2 (Schäfer et al., 2006; Schäfer et al., 2003). Once in the cytoplasm, it has been proposed that assembly factors physically block the association of the translation machinery by occupying functional sites on the 40 S subunit (Strunk et al., 2011). Structural insights into the architecture of pre-40S particles have previously been obtained through cryo-EM analysis, using preparations from both yeast and human cells (Strunk et al., 2011; Johnson et al., 2017; Larburu et al., 2016). In combination with RNA-protein crosslinking data, these structures have allowed the approximate positioning of most of the biogenesis factors on the late pre-40S particles (Strunk et al., 
2011; Granneman et al., 2010). However, in contrast to recent higher resolution structures obtained for the early 60S intermediates (Greber, 2016), no late pre-40S structures with atomic resolution are available. Accordingly, detailed insight into the molecular interactions of the RBFs and the conformation of the pre-rRNA in late $40 \mathrm{~S}$ pre-ribosomes was lacking.

\section{Results and discussion}

To gain a better understanding of the small ribosomal subunit biogenesis on a molecular level we purified late pre-40S particles via the well-defined biogenesis factor Ltv1 (Schäfer et al., 2006; Johnson et al., 2017), using Ltv1-Flag-TEV-ProteinA (FTpA) as bait (Figure 1-figure supplement 1A). With this strategy, we obtained a high yield of homogeneous pre-40S particles, which were used for single particle cryo-EM (Figure 1-figure supplement 1B-C). After classification we obtained a major class containing the stably bound RBFs Enp1, Tsr1, Rio2 and Pno1 (Figure 1-figure supplement 1D) but lacking a number of late binding ribosomal proteins (RACK1, uS10, uS14, eS10, eS26 and uS3) (Ferreira-Cerca et al., 2007). This main class could be refined to an average resolution of $3.6 \AA$, with the local resolution ranging from $3.5 \AA$ in the core to approximately $8 \AA$ for flexible regions (Figure 1-figure supplement 2). We built atomic models for Tsr1 and Pno1, and were able to model Enp1 and Rio2 on a secondary structure level (Figure 1A and Figure 1-figure supplements 2-3). In addition, the structure of the pre-18S rRNA revealed very distinct conformational differences, as compared to the mature state (Heuer et al., 2017), of functionally important regions including all three tRNA binding sites ( $A, P$ and $E$ ) and the entire mRNA path. We found that two major rRNA condensation steps still have to happen for these sites to mature: one in the head/ beak region and the other in the central region of the 18S rRNA (Figure 1B).

The characteristic tertiary structure of the head rRNA (h28 to h43) is mainly determined by threeway junctions (Mohan et al., 2014). We observe that in the pre-18S rRNA only one such junction is not yet formed, namely that which connects h34, h35 and h38 (Figure 1C and Figure 1-figure supplement 4A). It joins three blocks of rRNA, which all contain parts of functionally important regions: one block contains h33 of the beak and h34, a central element in the formation of the A-site decoding center, while the block comprising h29-h32 and h38-h42 contains key residues for mRNA binding and accommodation of anticodon-arms for all three tRNAs. The third block (h35-h37) contains h36, which forms important tertiary interactions between head and body and is part of the central region of the 18S rRNA (Wimberly et al., 2000) (see below). Due to the absence of this junction, these blocks are shifted relative to each other and relative to the body, preventing the formation of the actives sites. Notably, formation of this junction requires the incorporation and stabilisation of the late associating ribosomal proteins uS3, uS10 and uS14 (Lescoute and Westhof, 2006), which are absent from the pre-40S particle (Figure 2-figure supplement 1).

The central region of the $18 \mathrm{~S}$ rRNA comprises the central pseudoknot (CPK), a universally conserved structure that connects the head with the body via h28, h1 and h2. It provides a core structure around which major parts of the active A- and P-sites form, with the most central being h44 and h28. The tip of h44 contains two universally conserved adenosine bases (A1755/A1755, A1492/ A1493 in E. coli) critical for mRNA decoding (Ogle et al., 2003) and the 'neck helix' h28 provides a hinge for head rotation, which is crucial for tRNA movement during elongation (Mohan et al., 2014; Korostelev et al., 2008). Formation of the CPK is a major structural landmark and we observe that, unlike in the 90S, the CPK is fully folded and the contact with the head (h36) has been established (Figure 1-figure supplement $4 \mathrm{~B}-\mathrm{C}$ ). In contrast, we observe that the top of h44 is not yet basepaired, and the linker of h44 with h28 and h45 remains highly flexible. Notably, this linker forms major parts of the $A$ and $P$ sites in the mature state (Figure 1D and Figure 1-figure supplement $4 B$ ). Moreover, h44 is repositioned outwards relative to its mature position and h28 is tilted by 12 degrees in the direction of the beak (Figure 1-figure supplement 4B-C). Collectively, we observe that the pre-18S rRNA is still in a non-functional immature state since all elements forming the active decoding and mRNA interaction sites are prevented from adopting their mature fold (Figure 2).

We next investigated the role that the RBFs play regarding the immature pre-18S rRNA conformation. The first RBF, Tsr1, shares a similar domain architecture (I-IV) to several translational GTPases with an additional N-terminal extension, which was unresolved in previous studies (Johnson et al., 2017; McCaughan et al., 2016). Tsr1 mainly binds to the region which forms the universal translation factor binding site on the small subunit. Tsr1 contacts the junction of h5 - h15 and uS12 via its 
A
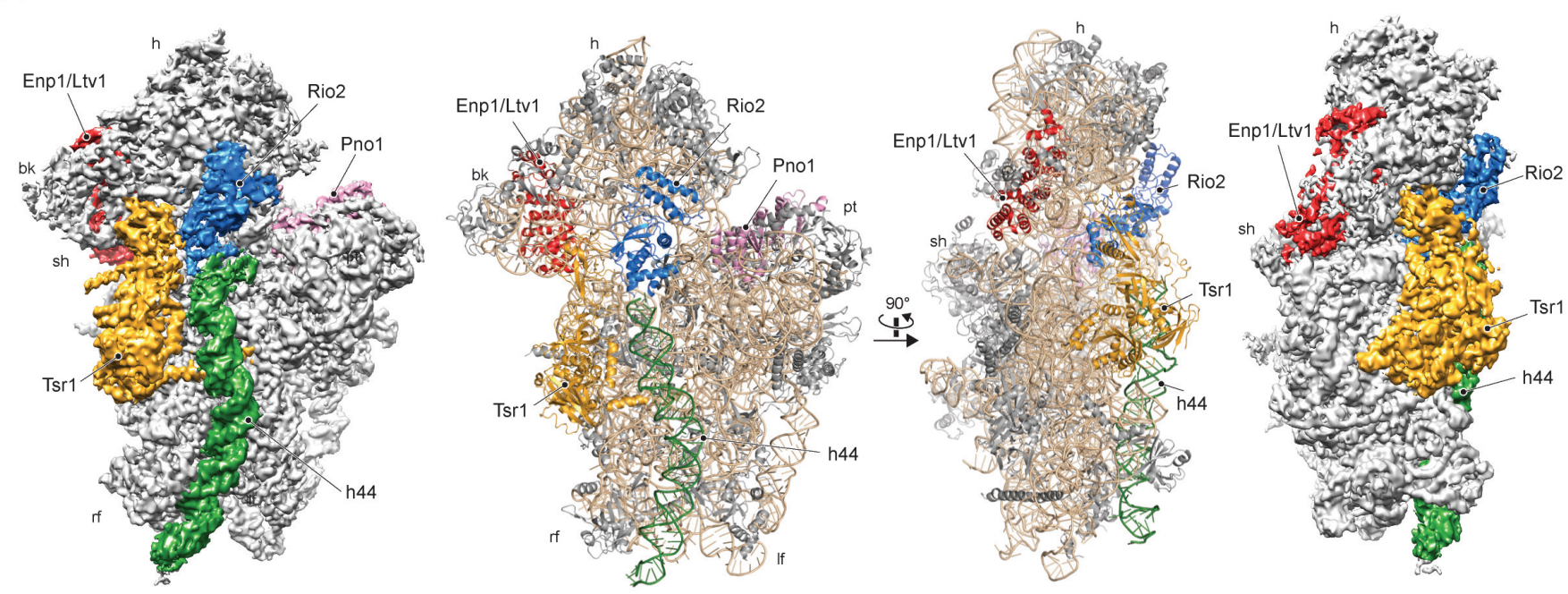

B
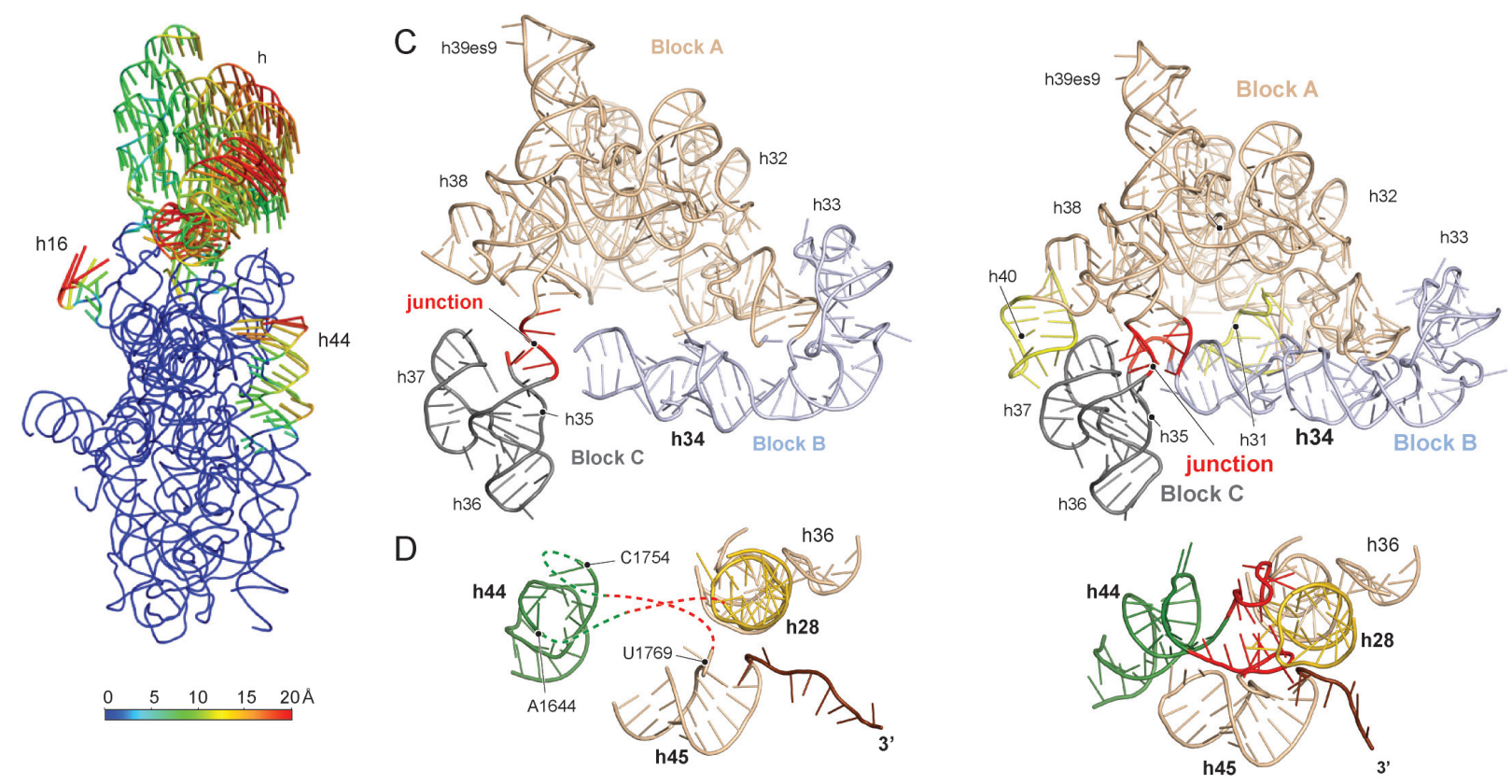

Figure 1. Structure of the pre-40S particle and conformation of the $18 \mathrm{~S}$ rRNA.. (A) $3.6 \AA$ cryo-EM reconstruction and molecular model of the pre-40S complex containing Enp1/Ltv1, Tsr1, Rio2 and Pno1 (B) Conformational transition of the 18S rRNA from the pre-mature to the mature state represented by vectors (superimposed on the $40 \mathrm{~S}$ body). (C) Condensation of the head-forming rRNA on the h34/35/38 three-way junction from pre- (left) to mature state (right). Block A (h29-h32, h38-h42) mostly resembles the mature state and served as the moiety for superposition. Relative to block A, blocks B (h34 and 33) and C (h35-h37) are still shifted, since the three-way junction linking the blocks is not yet established. The connection of h40 to h37 and the loop of h31 are not established in the pre-state. (D) View focusing on the the linker between h44 with h28 and h45 from the central region of $18 \mathrm{~S}$ rRNA. In the pre-state (left).he linker region as well as parts of h44 are unfolded and h28 (yellow) is tilted compared to the mature state (right). DOI: https://doi.org/10.7554/eLife.30189.002

The following figure supplements are available for figure 1:

Figure supplement 1. Purification and cryo-EM of the pre-40S complex.

DOI: https://doi.org/10.7554/eLife.30189.003

Figure supplement 2. Assessment of resolution and model quality of the cryo-EM structure.

DOl: https://doi.org/10.7554/eLife.30189.004

Figure supplement 3. Fitting of the RBFs.

DOI: https://doi.org/10.7554/eLife.30189.005

Figure 1 continued on next page 
Figure 1 continued

Figure supplement 4. Conformation and flexibility of the pre-18S rRNA

domains I and III (Figure 3A). Specific interactions are established with h17 and to the N-terminal tail of eS30, which is yet to adopt its mature position (Figure 4-figure supplement 1). Further, domain IV of Tsr1 links the body with the immature head contacting h30 to h32, as well as the shifted h34. Notably, these rRNA structures are contacted from the opposite side by Enp1/Ltv1 (see below), resulting in the coordinated stabilization of the ribosomal beak in its immature conformation.

Due to high local resolution, we were able to build the $\mathrm{N}$-terminal part of Tsr1. It forms a $35 \AA$ long $\alpha$-helix, which pierces through the ribosome between h5 and h44. By reaching further it touches h11-h12 (Figure 3A-B), thus serving as a distance enforcing wedge for h44. Thereby, via a long distance effect, Tsr1 keeps the linker connecting h44 with h28 and h45 unfolded and immaturely positioned. To assess the functional significance of the interaction of the N-terminal helix of Tsr1 and h44 we generated reverse charge mutants (R54D, K55D, K57D and K59D) where combinations of 3 or more substitutions indeed resulted in a slow growth phenotype (Figure 3C). All mutants showed the same nuclear localization, but a decrease in association with pre-ribosomes as compared to wildtype Tsr1 (wt) (Figure 4-figure supplement 1A-B). These data suggest that the $\mathrm{N}$-terminus of Tsr1 is important for both, the stabilization of h44 in its immature conformation and the association of Tsr1 with pre-ribosomes. We also assessed the consequences of abolishing the interaction between domain IV of Tsr1 and the head of the pre-40S by removing domain IV. This mutant no longer supported yeast cell growth but continued to interact with pre-ribosomal particles (Figure 4-figure supplement 1C-D). We therefore propose that domain IV is not necessary for the association of Tsr1 with the pre-ribosome, but rather plays an important role to stabilize the pre-40S head in its immature conformation.

Enp1 is one of the few assembly factors that is already present in the $90 \mathrm{~S}$ particle and remains associated until the integration of uS3 during late pre-40S biogenesis in the cytoplasm (Kornprobst et al., 2016; Schäfer et alı, 2003). We observe Enp1 binding to the tip of the bent h16 near the mRNA entry site (Figure 3D, 4A). From there it reaches over to bind h32 - h34, thus keeping the head in its immature conformation together with Tsr1. Notably, we observe that Enp1 binds the same rRNA elements as in the 90S (Kornprobst et al., 2016; Sun et al., 2017; ChakerMargot et al., 2017), further suggesting an early stabilization of the ribosomal beak. Ltv1, Enp1 and Rps3 are known to form a stable protein complex (Schäfer et al., 2006). Most likely due to the absence of Rps3 in our structure, only the extra density on top of Enp1 likely corresponds to its interaction partner Ltv1, which is in agreement with previous structural studies (Strunk et al., 2011; Larburu et al., 2016). The binding site of Enp1/Ltv1 occupies the position of the as yet unincorporated protein eS10 (Figure 4A) and explains Enp1/Ltv1's described role in facilitating uS3 integration at an adjacent site (Schäfer et al., 2006). It further suggests a role for Enp1 in the maturation of h34 and the h34-h35-h38 three-way junction.

Rio2 a RBF conserved in all archaea and eukaryotes (Geerlings et al., 2003; Vanrobays et al., 2003; Schäfer et alı, 2003), is an essential serine kinase required for 40S maturation. It binds the pre-40S at the $A$ and $P$ site region with all three domains (Figure $3 E$ ). The $\mathrm{N}$-terminal winged-helixturn-helix motif (wHTH) contacts the tip of h18, which forms the 'latch' for the mRNA in the mature 40S together with uS3, h34 and uS12 (Schluenzen et al., 2000). The two-lobed kinase domains of Rio2, K1 and K2, are positioned close to h28 whereby $\mathrm{K} 1$ contacts the region, which serves as a hinge during the $40 \mathrm{~S}$ head rotation (Mohan et al., 2014). Rio2's K1 also contacts the $40 \mathrm{~S}$ head via h30 at a position close to domain IV of Tsr1. Finally, K2 contacts h29 and h42, which forms the P-site for binding the (initiation) tRNA in the mature ribosome.

We further identified Pno1, a factor that together with the endonuclease Nob1 controls one of the final events of $40 \mathrm{~S}$ biogenesis, the maturation of the 18S rRNA through cleavage of the $3^{\prime}$-end at cleavage site D (Lamanna and Karbstein, 2009; Lamanna and Karbstein, 2011). This cleavage event is believed to be regulated by Pno1 (Vanrobays et al., 2004), which belongs to the family of single-stranded RNA binding proteins with $\mathrm{KH}$-domains. It is located on the platform of the pre$40 \mathrm{~S}$ (Figure 5A) where it interacts with uS11/uS1, the tilted rRNA h28, h45 and the 3'-end of the 


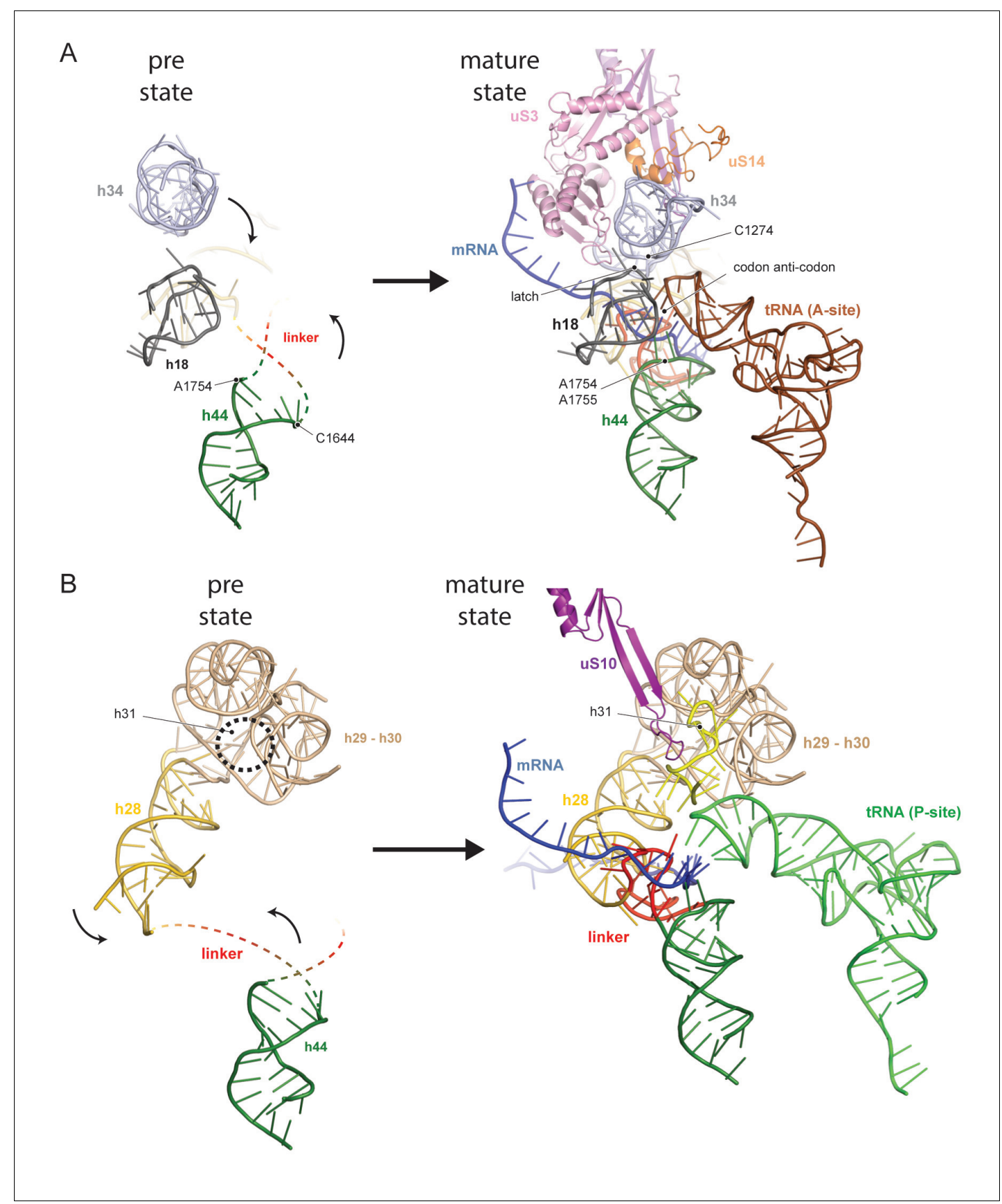

Figure 2. Comparison between pre- and native $40 \mathrm{~S}$ focusing on the active sites. (A) View focusing on the mRNA entry and A-site. The A-site is composed of $\mathrm{h} 18, \mathrm{~h} 34$ and $\mathrm{h} 44$, where in a translating ribosome the anticodon-loop of a A-tRNA is bound and the mRNA enters the 40S via the latch structure formed between h18, h34 and uS3. In the pre-40S, uS3 is absent and h34 is displaced. Moreover, h44 is shifted and its tip including the decoding adenines A1754 and A1755 is unfolded. (B) View focusing on the mRNA exit and the P-site. The P-site is composed of h24 (not shown), h28, h29 and h31 as well as the linker between h44 and h45. In the pre-40S this linker is delocalized and h28 is shifted. Moreover, the tip of h31 which is stabilized by uS10 in the mature state and binds the P-site tRNA in a translating ribosome is not folded in the pre-state.

DOI: https://doi.org/10.7554/eLife.30189.007

The following figure supplement is available for figure 2 :

Figure supplement 1. Structure and environment of the native h34-h35-h38 three-way junction. 


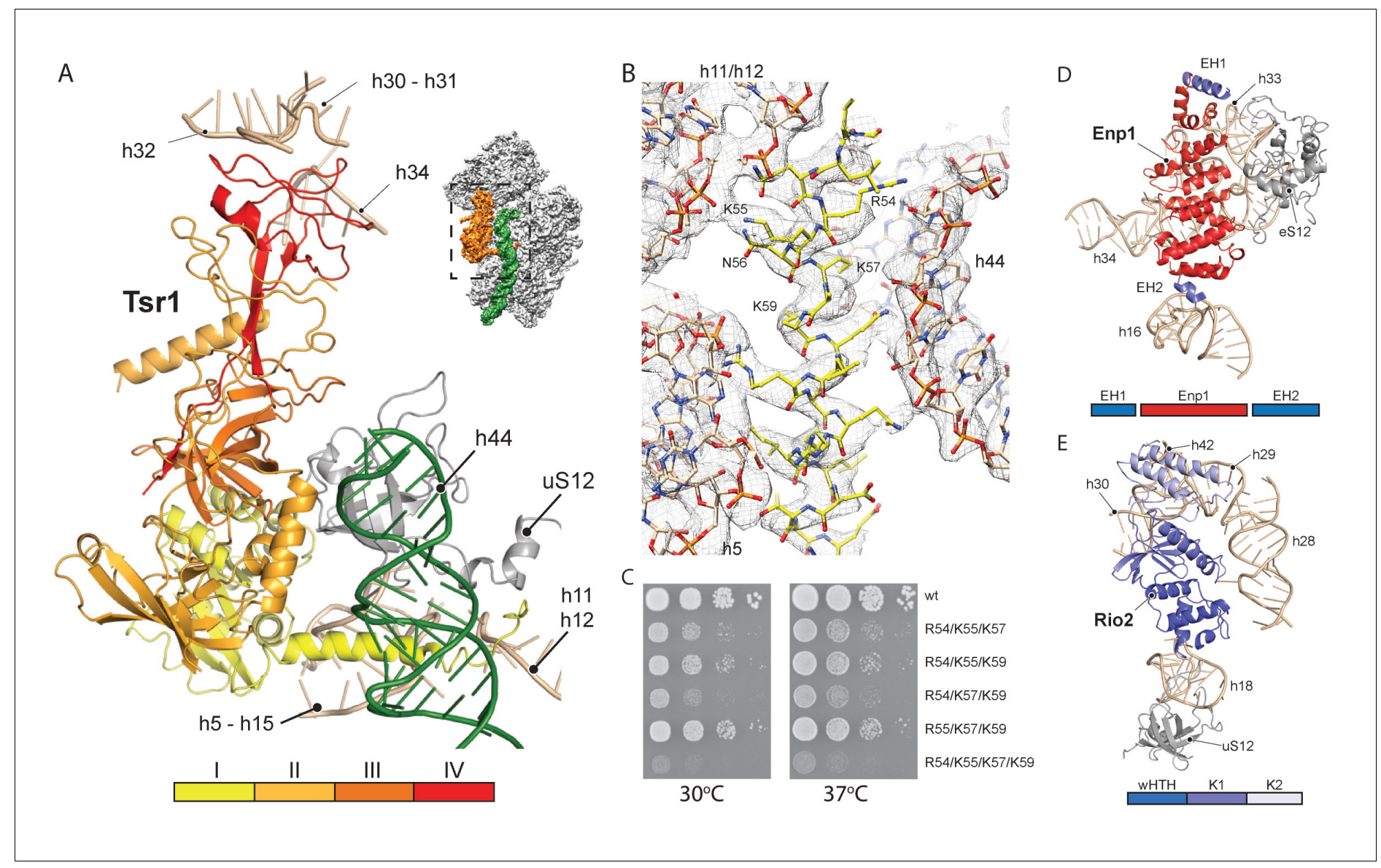

Figure 3. Interactions of Tsr1, Enp1/Ltv1 and Rio2 with the pre-40S. (A) Tsr1 binds the 40S body (h5-h15, h17 and uS12) via domains I and III, the 40S head (h30-h31, h32 and h34) via domain IV. The N-terminal $\alpha$-helix of Tsr1 intercalates between h44, h5 and h11-h12. (B) Model of the Tsr1 Nterminal $\alpha$-helixfitted into density low-pass filtered at $3.6 \AA$. (C) Growth analysis of wt Tsr1 and reverse-charge point mutations in residues interacting with h44 (R54D, K55D, K57D and K59D). Constructs were transformed into a Tsr1 shuffle strain and selected on SDC + FOA plates. Strains were spotted in 10-fold serial dilution on YPD plates and incubated for 2 days at the indicated temperatures. Different temperatures were used to assess if the growth defect observed at 30 degrees was enhanced at higher temperatures. (D) Enp1 binds to h33, h34 and eS12 and to the kinked h16; EH = extra helix for Enp1 or Ltv1. (E) Rio2 binds the 40S body via its N-terminal winged-helix-turn-helix-domain (wHTH) (h18, uS12), and the 40S head via the twolobed kinase domain (K1/K2) (h28,h30). Moreover, K2 reaches into the P-site contacting h29, h42 and the region in between.

DOI: https://doi.org/10.7554/eLife.30189.009

The following figure supplement is available for figure 3 :

Figure supplement 1. Functional analyses of mutant forms of Tsr1.

DOI: https://doi.org/10.7554/eLife.30189.010

pre-18S rRNA. Importantly, in this position, Pno1 sterically hinders h28 from adopting its mature conformation and the binding of eS26 (Figure 4B). We were able to follow the 3' rRNA end, bound by Pno1, up to the pre-terminal base (U1799, D cleavage occurs after A1800) in molecular detail: A multitude of interactions is formed by three $\alpha$-helices of the $\mathrm{KH} 2$ domain of Pno1, which recognize the first two single-stranded bases (G1793 and A1794) as well as the stem of h45 (Figure 5B) including the last base of the h44-h45 linker (U1769), which later will form a part of the active $\mathrm{P}$ - and mRNA binding sites. Thus Pno1, like Tsr1 and Rio2, prevents compaction of the central region of $18 \mathrm{~S}$ rRNA.

Like other members of the KH family (Nicastro et alı, 2015), Pno1-KH2 uses its hallmark GXXGRNA binding motif to position four nucleobases (residues 1795-1798) in a hydrophobic pocket (Figure 5C-D). Interestingly, KH1, which lacks the signature GXXG sequence in yeast (Woolls et al., 2011), also contributes to $3^{\prime}$-binding and contacts the terminal 18S rRNA bases 1797-1799 via its $\alpha$ helix h1. Sequence alignments for the $3^{\prime}$-end revealed not only that the UCAU sequence - 


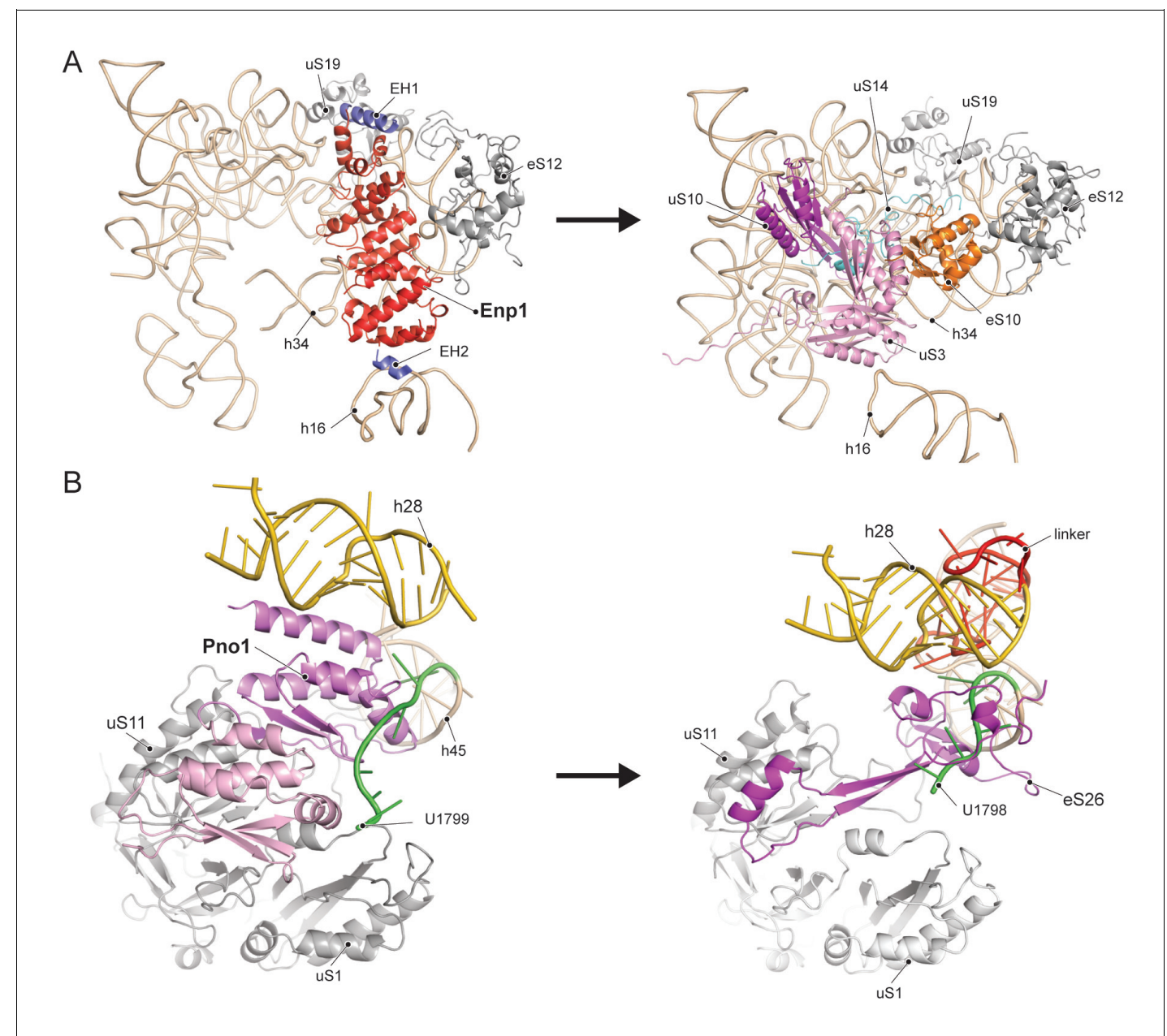

Figure 4. Comparison of RBF binding sites in the premature and the mature 40S. (A) Enp1/Ltv1 binds near the mRNA entry channel and connects the shoulder (h16) with the beak (h33, h34 and eS12). It occupies the position of the eS10. Moreover, uS3, uS10 and uS14 are not incorporated into the pre$40 \mathrm{~S}$ particle. Note that h16 is in a bent conformation compared to the mature state. (B) Pno1 binds at the platform of the pre-40S contacting uS1, uS11, the kinked h28 as well as h45 and the $3^{\prime}$-end of 18S rRNA. It thereby occupies the position of eS26, which binds the rearranged $3^{\prime}$ end in the mature state.

DOI: https://doi.org/10.7554/eLife.30189.011

The following figure supplement is available for figure 4:

Figure supplement 1. Binding of Tsr1 to h17 and eS30.

DOI: https://doi.org/10.7554/eLife.30189.012

that contactes $\mathrm{KH} 2$ - is conserved from yeast to humans, but also the surrounding bases up to the D-cleavage site. This suggests that specific binding of Pno1 and positioning of the 3'-end in a distinct conformation may be a universal feature of eukaryotic ribosome maturation. Notably, Pno1 is in an ideal location to sense any further maturation of $18 \mathrm{~S}$ rRNA, in particular conformational changes of the close-by h28, which may allow Pno1 to productively present the D-site for cleavage by the neighbouring endonuclease Nob1. In addition, Pno1 may protect the $3^{\prime}$ end against further cleavage until the small ribosomal subunit is fully matured.

In conclusion, we have discovered that the collective association of a few ribosome biogenesis factors on the late pre-40S ribosome regulates final rRNA folding steps at functionally important sites, in particular at the decoding centre. It appears that the role of these factors is to temporarily maintain the $40 \mathrm{~S}$ subunit in a translationally incompetent state during ribosome biogenesis, preventing premature substrate interaction or entry into cycles of translation, which would be error-prone and potentially harmful to the cell. We envision that removal of biogenesis factors and the maturation of these regions are inter-dependent and coordinated processes. Conditional stepwise removal 


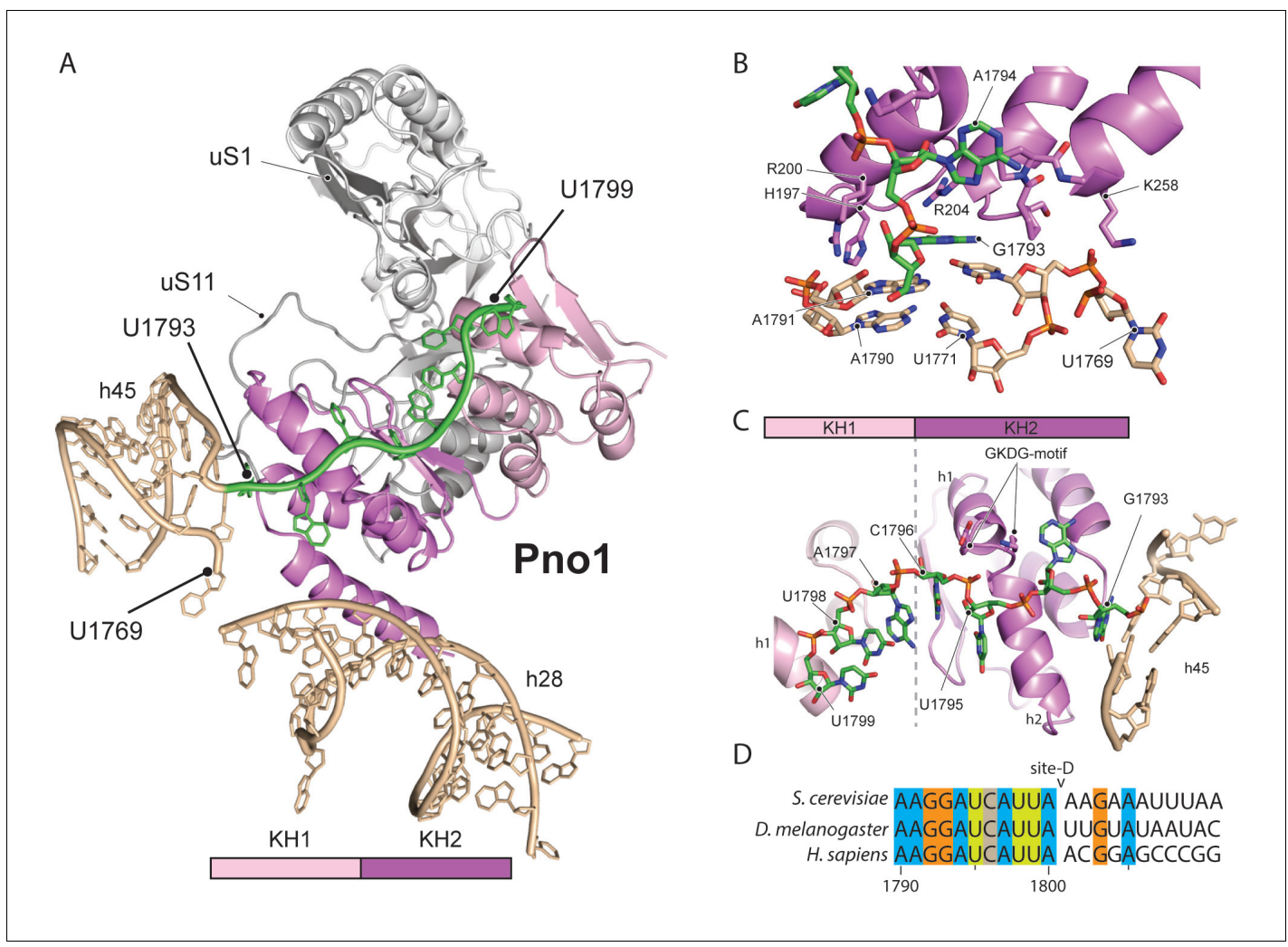

Figure 5. Molecular interactions of Pno1. (A) Pno1 binds uS11/uS1, h45, the tilted h28 and the 3' end (green) at the platform. (B) View focusing on the molecular interactions of KH2 with h45. Three C-terminal $\alpha$-helices recognize the stem of h45 (A1791:U1770 and A1790:U1769), upon which G1793 is stacked. Specifically, Arg204 contacts G1793 and Arg200 together with His197 the backbone of the 3' strand (A1791 and A1790). The loop between two of the C-terminal helices of Pno1 (Gly253-Lys258) contacts U1770 and U1769 of the 5'-strand of h45. This loop also participates in binding of the first single-stranded base A1794 which is sandwiched between Pro256 and the GKDG-loop of KH2 (C) View focusing on the molecular interactions between Pno1 and the 3' rRNA end. Both KH domains interact with the 3'-end rRNA residues (G1793-U1799) which lead up to the D-cleavage site. The GKDGloop of $\mathrm{KH} 2$ positions four nucleobases (U1795-U1798) close to its hydrophobic pocket and KH1 contacts the terminal bases (A1797-U1799) via h1 (D) Sequence alignment for eukaryotic 3' rRNA ends showing conservation up to the D-cleavage site.

DOI: https://doi.org/10.7554/eLife.30189.013

of these biogenesis factors may therefore serve as checkpoints that ensure the structural integrity of the ribosomal subunit, and thereby fitness for translation.

\section{Materials and methods}

Key resource table

\begin{tabular}{|c|c|c|c|c|}
\hline Reagent type (species) or resource & Designation & Source or reference & Identifiers & Additional information \\
\hline $\begin{array}{l}\text { strain, strain background } \\
\text { (Saccharomyces cerevisiae) }\end{array}$ & Ltv1-FTpA;Tsr1shuffle & This paper & NA & $\begin{array}{l}\text { Ltv1-FTpA-Genomic copy of Ltv1 } \\
\text { tagged at the c-terminus with } \\
\text { FLAG-TEV-proteinA tag; Tsr1 } \\
\text { shuffle- genomic copy of Tsr1 } \\
\text { deleted and rescued with a } \\
\text { plasmid with a wild-type copy }\end{array}$ \\
\hline
\end{tabular}

Continued on next page 
Continued

Reagent type (species) or resource

genetic reagent (plasmids used for expression in

Saccharomyces cerevisiae)
Designation

YCplac111-Tsr1-FTpA

YCplac111-Tsr1 R54D,

K55D,K57D-FTpA;

YCplac111-Tsr1 R54D,

K55D,K59D-FTpA;

YCplac111-Tsr1 R54D,

K57D,K59D-FTpA;

YCplac111-Tsr1 K55D,

K57D,K59D-FTpA

YCplac111-Tsr1 R54D,

K55D,K57D,K59D-FTpA;

YCplac111-Tsr1-GFP;

YCplac111-Tsr1 R54D,

K55D,K57D,K59D-GFP;

YCplac111-

Tsr1DC86-FTpA

\begin{tabular}{|c|c|c|c|c|}
\hline software, algorithm & EM-TOOLS & TVIPS GmbH & NA & $\begin{array}{l}\text { http://www.tvips.com/ } \\
\text { imagingsoftware/em-tools/ }\end{array}$ \\
\hline software, algorithm & MotionCorr2.1 & $\begin{array}{l}\text { https://doi.org/ } \\
\text { 10.1038/nmeth.4193 }\end{array}$ & NA & $\begin{array}{l}\text { http://cryoem.ucsf.edu/ } \\
\text { software/driftcorr.html }\end{array}$ \\
\hline software, algorithm & GCTF & $\begin{array}{l}\text { https://doi.org/ } \\
\text { 10.1016/j.jsb.2015.11.003 }\end{array}$ & NA & $\begin{array}{l}\text { http://www.mrclmb. } \\
\text { cam.ac.uk/kzhang }\end{array}$ \\
\hline software, algorithm & Gautomatch & public & NA & $\begin{array}{l}\text { http://www.mrclmb. } \\
\text { cam.ac.uk/kzhang }\end{array}$ \\
\hline software, algorithm & Relion-2 & $\begin{array}{l}\text { https://doi.org/ } \\
\text { 10.7554/eLife.18722 }\end{array}$ & NA & $\begin{array}{l}\text { http://www2.mrclmb.cam.ac } \\
\text {.uk/relion/index.php }\end{array}$ \\
\hline software, algorithm & $\begin{array}{l}\text { Phenix suite } \\
\text { (phenix.real_space_- } \\
\text { refine, molprobity) }\end{array}$ & $\begin{array}{l}\text { Python-based Hierarchical } \\
\text { ENvironment for Integrated } \\
\text { Xtallography }\end{array}$ & RRID:SCR_014224 & https://www.phenix-online.org/ \\
\hline software, algorithm & $\begin{array}{l}\text { CCP4 (LIBG, } \\
\text { ProSMART, Refmac5, } \\
\text { COOT) }\end{array}$ & $\begin{array}{l}\text { Collaborative Computational } \\
\text { Project No. } 4 \text { Software } \\
\text { for Macromolecular } \\
\text { X-Ray Crystallography }\end{array}$ & RRID:SCR_007255 & http://www.ccp4.ac.uk/ \\
\hline software, algorithm & UCSF Chimera & $\begin{array}{l}\text { UCSF Resource for } \\
\text { Biocomputing, Visualization, } \\
\text { and Bioinformatics }\end{array}$ & RRID:SCR_004097 & http://www.cgl.ucsf.edu/chimera/ \\
\hline software, algorithm & Pymol & $\begin{array}{l}\text { PyMOL Molecular Graphics } \\
\text { System, Schrödinger, LLC }\end{array}$ & RRID:SCR_000305 & https://pymol.org/ \\
\hline
\end{tabular}

\section{Yeast strains and plasmids}

For affinity purification of pre-40S particles for EM analysis, endogenous Ltv1 was tagged in a DS1$2 \mathrm{~b}$ background (Nissan et al., 2002) at its C-terminus with a FLAG-Tev-protA (FTpA), as previously described (Longtine et al., 1998). All wt and mutant Tsr1-FLAG-Tev-protA or Tsr1-GFP constructs were expressed from plasmids under the control of the endogenous promoter. For Tsr1 affinity purification and localization studies, constructs were expressed in a BY4741 background. For growth analysis, constructs were transformed into a Tsr1 shuffle strain (in a BY4741 background), followed by selection on SDC + FOA. All constructs used in this study can be found in the key resource table.

\section{Affinity purification from yeast lysates}

Purifications of all FTpA-tagged bait proteins were performed in buffer containing $50 \mathrm{mM}$ Tris- $\mathrm{HCl}$ (pH 7.5), $100 \mathrm{mM} \mathrm{NaCl}, 1.5 \mathrm{mM} \mathrm{MgCl} 2,5 \%$ glycerol, $0.1 \% \mathrm{NP}-40$, and $1 \mathrm{mM} \mathrm{DTT}$. Cell lysates were prepared using a beadbeater (Fritsch), followed by centrifugation. Pre-equilibrated IgG Sepharose (GE) was added to the supernatant and incubated for $90 \mathrm{~min}$ at $4^{\circ} \mathrm{C}$. Extensive washing was followed by TEV cleavage and a second step of purification on anti-FLAG MS2-agarose beads. Beads were washed, and proteins were eluted using a buffer containing $50 \mathrm{mM}$ Tris- $\mathrm{HCl}(\mathrm{pH} 7.5), 100 \mathrm{mM} \mathrm{NaCl}$, $1.5 \mathrm{mM} \mathrm{MgCl} 2,1 \mathrm{mM}$ DTT, 3xFLAG peptide. For cryo-EM analysis the FLAG eluate was directly used. For Tsr1 purifications FLAG eluates were precipitated with TCA (10\% final) and resuspended in 
Table 1. EM data collection and refinement statistics.

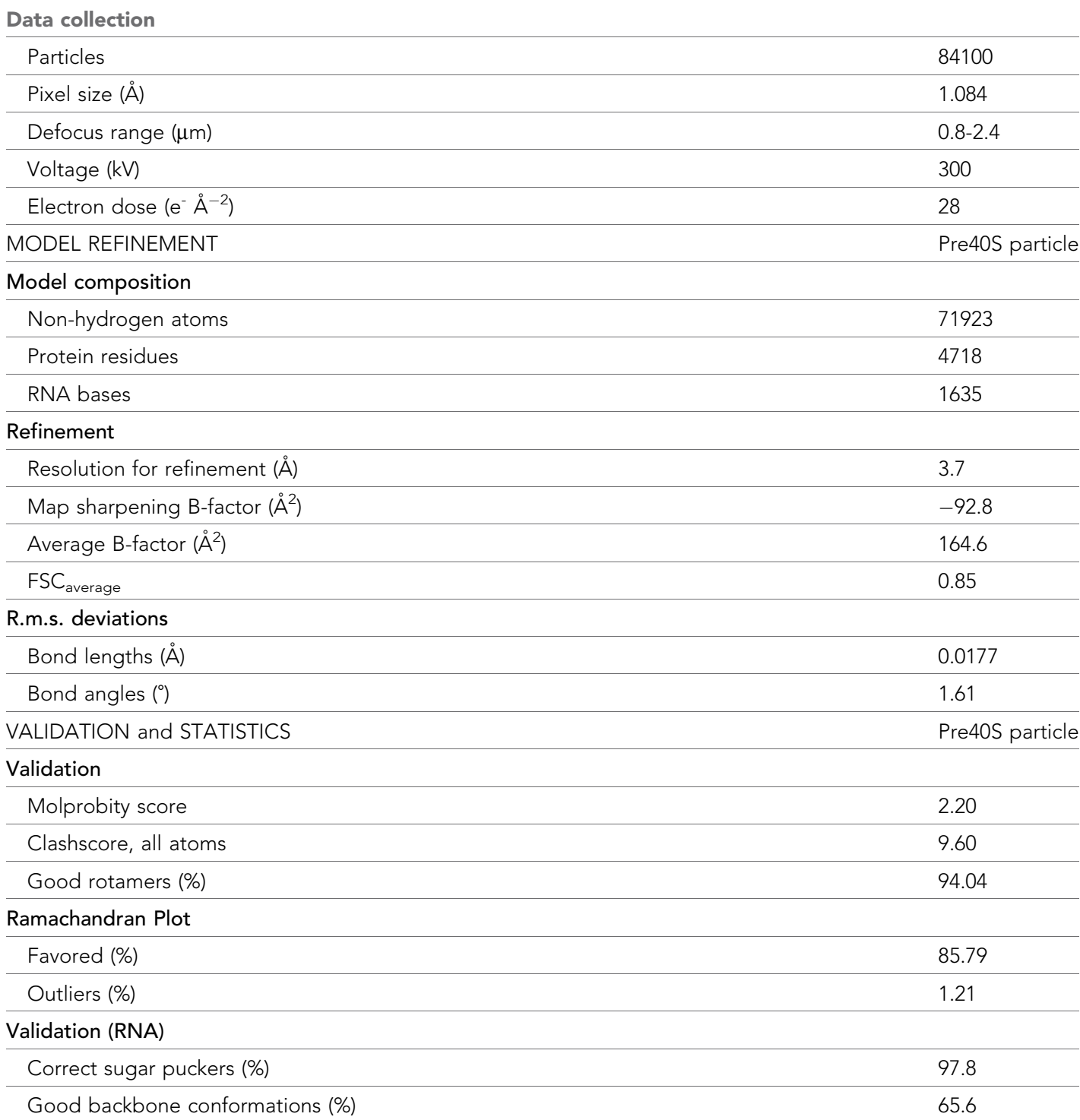

DOI: https://doi.org/10.7554/eLife.30189.014

SDS sample buffer. Proteins were separated on 4-12\% NuPAGE polyacrylamide gel and stained with colloidal Coomassie.

\section{Electron microscopy and image processing}

Freshly prepared samples were adjusted to $1.5 \mathrm{~A}_{260}$ (50 nM 40S ribosomes) and applied to Quantifoil R3/3 holey grids pre-coated with $2 \mathrm{~nm}$ carbon. Data was collected on a Titan Krios TEM (FEI Company) equipped with a Falcon II direct electron detector at $300 \mathrm{keV}$ under low dose conditions of about 2.4 e-/Å2 per frame for 10 frames in total using the software EM-TOOLS (TVIPS) and a defocus range of -0.8 to $-2.4 \mu \mathrm{m}$ at a pixel size of $1.08 \AA$. Original image stacks were summed up and corrected for drift and beam-induced motion at micrograph level using MotionCor2 (Zheng et al., 2017). The contrast transfer function parameters and resolution range of each micrograph were estimated by GCTF (Zhang, 2016). All 2D and 3D classifications and refinements were performed with RELION-2 (Kimanius et al., 2016) after automated particle picking by Gautomatch (http://www.mrc-Imb.cam.ac.uk/kzhang/). Two-dimensional reference-free classification was performed to screen for particle quality (Figure 1-figure supplement 1C), non-ribosomal particles as well as poorly resolved 2D classes were discarded. 266.800 particles from good classes were 
selected for 3D refinement using a mature $40 \mathrm{~S}$ ribosome as reference. We performed two subsequent rounds of $3 \mathrm{D}$ classification in order to enrich pre-40S complexes (Figure 1-figure supplement 1D). First, the whole dataset was classified into 7 classes: class 1 and 2 contained orientation biased $40 \mathrm{~S}$ ribosomes whereas classes 3 to 5 showed well-resolved $40 \mathrm{~S}$ ribosomes with strong extra densities for the RBFs. In addition, class 6 showed poorly resolved pre-40S ribosomes and class 7 displayed a pre-40S ribosome with a very flexible head domain. The classes 3 to 5 were joined for movie refinement and a second round of 3D classification (six classes). Here, class 1 displayed distorted density due to orientation bias, while class 2 and 3 showed a very strong density for the majority of RBFs. Class 4 displayed a less distorted volume than class 1 but showed an extra density emanating from the platform to the head, which is likely Nob1 (Strunk et al., 2011). In addition Class 5 showed weak densities for RACK1, uS3 and the Dim1(Johnson et al., 2017). The most promising classes 2 and 3 were joined for final refinement and used for further interpretation. This final volume contained 84.100 particles was refined to $3.6 \AA$ (FCS $=0.143$ ) according to the 'gold standard' criterion, corrected for the modulation transfer function of the Falcon two detector and sharpened by applying a negative B-factor automatically estimated by RELION-2. Local resolution was calculated from 3.5 to $8.0 \AA$ in steps of $0.5 \AA$ using ResMap (Kucukelbir et al., 2014).

\section{Model building}

For model building of the pre-40S subunit the structure of the mature $S$. cerevisiae $40 \mathrm{~S}$ post splitting complex was used as a template (PDB 5LL6 [Heuer et al., 2017]). Available structures of the biogenesis factors Rio2 (PDB 4GYG [Ferreira-Cerca et al., 2012]), Tsr1 (PDB 5IW7 [McCaughan et al., 2016]), Pno1 (PDB 5WYJ [Sun et al., 2017]) and Enp1 (PDB 5WYJ [Sun et al., 2017]) were first fitted as rigid bodies into the isolated and appropriately low-pass filtered electron densities using UCSF Chimera (Pettersen et al., 2004). After rough docking, and manual adjustments where needed, flexible fitting and jiggle fitting was applied in Coot (Brown et al., 2015; Emsley and Cowtan, 2004). Regions not present in the available structures were modelled de novo, where the local resolution of the map allowed it (for example Tsr1, Pno1). rRNA which could, due to flexibility not be modelled with sufficient reliability, was removed from the model. In order to identify the extra density that may correspond to Ltv1, we fitted the structure of Enp1 found in the 90S pre-ribosome into the density. This fit left two rod-like densities unexplained, which we designated as extra helices 1 and 2 (EH1 and EH2). We speculated that these extra densities are either Ltv1 or an as yet unidentified part of Enp1. All models were subsequently combined and subjected to real-space refinement using PHENIX (Adams et al., 2010). After PHENIX refinement, the model was further subjected to reciprocal space refinement in REFMAC v5.8 (Murshudov et al., 1997) using restraints generated by ProSMART and LIBG as previously shown (Brown et alı, 2015; Amunts et al., 2014). Because of the difference in local resolution and to avoid overfitting, h34, Enp1/Ltv1 and Rio2 were not subjected to REFMAC refinement. The final model was validated using MolProbity (Chen et al., 2010), summarized statistics are displayed in Table 1. Cross-validation against overfitting was performed as previously described (Amunts et alı, 2014; Fernández et alı, 2014) for both model refinements separately. Figures were created with the PyMOL Molecular Graphics System (Version 1.7.4, Schrödinger, LLC) and with UCSF Chimera.

\section{Accession codes}

The EM density map is deposited in the 3D-EM database (EMD-3886) and the coordinates of the EM-based model is deposited in the Protein Data Bank (PDB-6EML).

\section{Acknowledgements}

The authors thank S Lange, H Sieber, M Gnädig and S Griesel for technical assistance; M Thoms for the Ltv1-FTpA strain; L Kater for valuable assistance with high-performance computing; J Cheng for fruitful discussions. We thank the Leibniz-Rechenzentrum Munich (LRZ) for providing computational services and support. 


\section{Additional information}

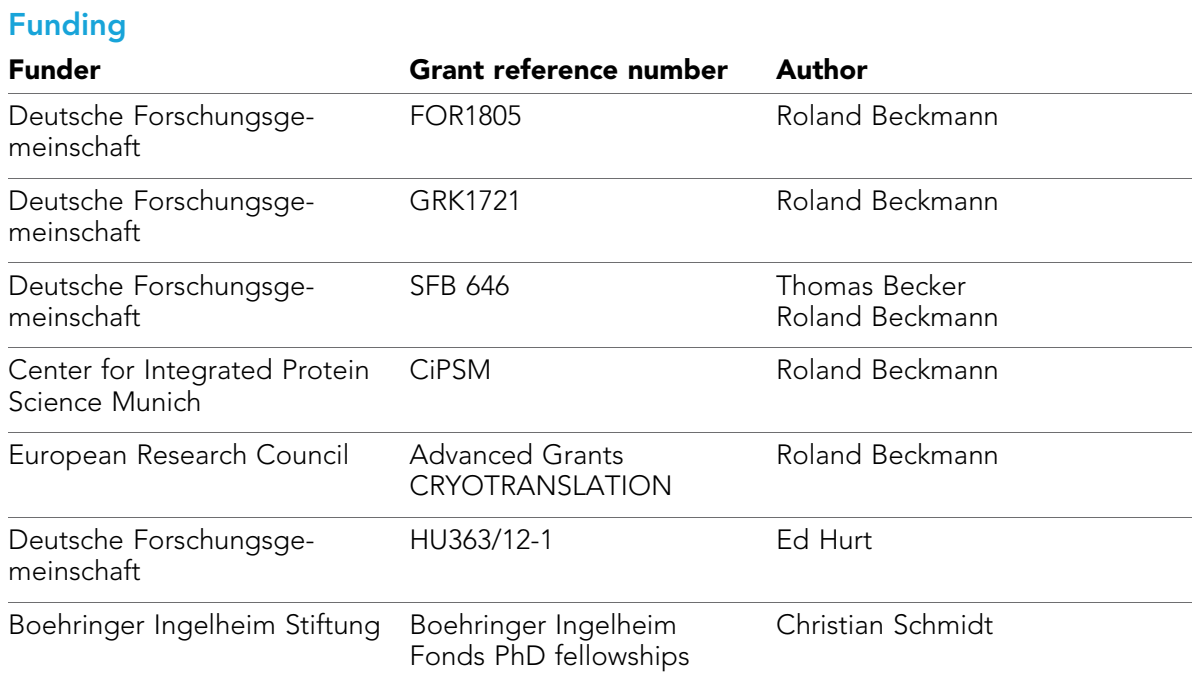

The funders had no role in study design, data collection and interpretation, or the decision to submit the work for publication.

Author contributions

André Heuer, Data curation, Formal analysis, Validation, Investigation, Visualization, Methodology, Writing-original draft, Writing-review and editing; Emma Thomson, Resources, Data curation, Validation, Writing-review and editing; Christian Schmidt, Validation, Writing-review and editing; Otto Berninghausen, Data curation; Thomas Becker, Conceptualization, Supervision, Writing-original draft, Writing-review and editing; Ed Hurt, Conceptualization, Funding acquisition, Writingreview and editing; Roland Beckmann, Conceptualization, Supervision, Funding acquisition, Validation, Writing—original draft, Project administration, Writing—review and editing

Author ORCIDs

André Heuer, (iD https://orcid.org/0000-0001-6144-4316

Thomas Becker,(DiD http://orcid.org/0000-0001-8458-2738

Roland Beckmann,(iD) https://orcid.org/0000-0003-4291-3898

Decision letter and Author response

Decision letter https://doi.org/10.7554/eLife.30189.016

Author response https://doi.org/10.7554/eLife.30189.017

\section{Additional files}

Supplementary files

- Transparent reporting form

DOI: https://doi.org/10.7554/eLife.30189.015

\section{References}

Adams PD, Afonine PV, Bunkóczi G, Chen VB, Davis IW, Echols N, Headd JJ, Hung LW, Kapral GJ, GrosseKunstleve RW, McCoy AJ, Moriarty NW, Oeffner R, Read RJ, Richardson DC, Richardson JS, Terwilliger TC, Zwart PH. 2010. PHENIX: a comprehensive Python-based system for macromolecular structure solution. Acta Crystallographica Section D Biological Crystallography 66:213-221. DOI: https://doi.org/10.1107/ S0907444909052925, PMID: 20124702

Amunts A, Brown A, Bai XC, Llácer JL, Hussain T, Emsley P, Long F, Murshudov G, Scheres SHW, Ramakrishnan V. 2014. Structure of the yeast mitochondrial large ribosomal subunit. Science 343:1485-1489. DOI: https:// doi.org/10.1126/science.1249410, PMID: 24675956 
Brown A, Long F, Nicholls RA, Toots J, Emsley P, Murshudov G. 2015. Tools for macromolecular model building and refinement into electron cryo-microscopy reconstructions. Acta Crystallographica Section D Biological Crystallography 71:136-153. DOI: https://doi.org/10.1107/S1399004714021683, PMID: 25615868

Chaker-Margot M, Barandun J, Hunziker M, Klinge S. 2017. Architecture of the yeast small subunit processome. Science 355:eaal1880-1889. DOI: https://doi.org/10.1126/science.aal1880, PMID: 27980088

Chen VB, Arendall WB, Headd JJ, Keedy DA, Immormino RM, Kapral GJ, Murray LW, Richardson JS, Richardson DC. 2010. MolProbity: all-atom structure validation for macromolecular crystallography. Acta Crystallographica Section D Biological Crystallography 66:12-21. DOI: https://doi.org/10.1107/S0907444909042073, PMID: 20057044

Dragon F, Gallagher JE, Compagnone-Post PA, Mitchell BM, Porwancher KA, Wehner KA, Wormsley S, Settlage RE, Shabanowitz J, Osheim Y, Beyer AL, Hunt DF, Baserga SJ. 2002. A large nucleolar U3 ribonucleoprotein required for 18S ribosomal RNA biogenesis. Nature 417:967-970. DOI: https://doi.org/10.1038/nature00769, PMID: 12068309

Emsley P, Cowtan K. 2004. Coot: model-building tools for molecular graphics. Acta Crystallographica. Section D, Biological Crystallography 60:2126-2132. DOI: https://doi.org/10.1107/S0907444904019158, PMID: 15572765

Fernández IS, Bai XC, Murshudov G, Scheres SH, Ramakrishnan V. 2014. Initiation of translation by cricket paralysis virus IRES requires its translocation in the ribosome. Cell 157:823-831. DOI: https://doi.org/10.1016/j. cell.2014.04.015, PMID: 24792965

Ferreira-Cerca S, Pöll G, Kühn H, Neueder A, Jakob S, Tschochner H, Milkereit P. 2007. Analysis of the in vivo assembly pathway of eukaryotic 40S ribosomal proteins. Molecular Cell 28:446-457. DOI: https://doi.org/10. 1016/j.molcel.2007.09.029, PMID: 17996708

Ferreira-Cerca S, Sagar V, Schäfer T, Diop M, Wesseling AM, Lu H, Chai E, Hurt E, LaRonde-LeBlanc N. 2012. ATPase-dependent role of the atypical kinase Rio2 on the evolving pre-40S ribosomal subunit. Nature Structural \& Molecular Biology 19:1316-1323. DOI: https://doi.org/10.1038/nsmb.2403, PMID: 23104056

Geerlings TH, Faber AW, Bister MD, Vos JC, Raué HA. 2003. Rio2p, an evolutionarily conserved, low abundant protein kinase essential for processing of 20 S Pre-rRNA in Saccharomyces cerevisiae. Journal of Biological Chemistry 278:22537-22545. DOI: https://doi.org/10.1074/jbc.M300759200, PMID: 12690111

Grandi P, Rybin V, Bassler J, Petfalski E, Strauss D, Marzioch M, Schäfer T, Kuster B, Tschochner H, Tollervey D, Gavin AC, Hurt E. 2002. 90 S pre-ribosomes include the 35S pre-rRNA, the U3 snoRNP, and 40S subunit processing factors but predominantly lack 60S synthesis factors. Molecular Cell 10:105-115. DOI: https://doi. org/10.1016/S1097-2765(02)00579-8, PMID: 12150911

Granneman S, Petfalski E, Swiatkowska A, Tollervey D. 2010. Cracking pre-40S ribosomal subunit structure by systematic analyses of RNA-protein cross-linking. The EMBO Journal 29:2026-2036. DOI: https://doi.org/10. 1038/emboj.2010.86, PMID: 20453830

Greber BJ. 2016. Mechanistic insight into eukaryotic 60S ribosomal subunit biogenesis by cryo-electron microscopy. RNA 22:1643-1662. DOI: https://doi.org/10.1261/rna.057927.116, PMID: 27875256

Henras AK, Plisson-Chastang C, O'Donohue MF, Chakraborty A, Gleizes PE. 2015. An overview of pre-ribosomal RNA processing in eukaryotes. Wiley Interdisciplinary Reviews: RNA 6:225-242. DOI: https://doi.org/10.1002/ wrna.1269, PMID: 25346433

Heuer A, Gerovac M, Schmidt C, Trowitzsch S, Preis A, Kötter P, Berninghausen O, Becker T, Beckmann R, Tampé R. 2017. Structure of the 40S-ABCE1 post-splitting complex in ribosome recycling and translation initiation. Nature Structural \& Molecular Biology 24:453-460. DOI: https://doi.org/10.1038/nsmb.3396, PMID: 2 8368393

Johnson MC, Ghalei H, Doxtader KA, Karbstein K, Stroupe ME. 2017. Structural Heterogeneity in Pre-40S Ribosomes. Structure 25:329-340. DOI: https://doi.org/10.1016/j.str.2016.12.011, PMID: 28111018

Kimanius D, Forsberg BO, Scheres SH, Lindahl E. 2016. Accelerated cryo-EM structure determination with parallelisation using GPUs in RELION-2. eLife 5:e18722. DOI: https://doi.org/10.7554/eLife.18722, PMID: 27 845625

Kornprobst M, Turk M, Kellner N, Cheng J, Flemming D, Koš-Braun I, Koš M, Thoms M, Berninghausen O, Beckmann R, Hurt E. 2016. Architecture of the 90S Pre-ribosome: A Structural View on the Birth of the Eukaryotic Ribosome. Cell 166:380-393. DOI: https://doi.org/10.1016/j.cell.2016.06.014, PMID: 27419870

Korostelev A, Ermolenko DN, Noller HF. 2008. Structural dynamics of the ribosome. Current Opinion in Chemical Biology 12:674-683. DOI: https://doi.org/10.1016/j.cbpa.2008.08.037, PMID: 18848900

Kucukelbir A, Sigworth FJ, Tagare HD. 2014. Quantifying the local resolution of cryo-EM density maps. Nature Methods 11:63-65. DOI: https://doi.org/10.1038/nmeth.2727, PMID: 24213166

Lamanna AC, Karbstein K. 2009. Nob1 binds the single-stranded cleavage site D at the $3^{\prime}$-end of 18S rRNA with its PIN domain. PNAS 106:14259-14264. DOI: https://doi.org/10.1073/pnas.0905403106, PMID: 19706509

Lamanna AC, Karbstein K. 2011. An RNA conformational switch regulates pre-18S rRNA cleavage. Journal of Molecular Biology 405:3-17. DOI: https://doi.org/10.1016/j.jmb.2010.09.064, PMID: 20934433

Larburu N, Montellese C, O’Donohue MF, Kutay U, Gleizes PE, Plisson-Chastang C. 2016. Structure of a human pre-40S particle points to a role for RACK1 in the final steps of 18S rRNA processing. Nucleic Acids Research 44:8465-8478. DOI: https://doi.org/10.1093/nar/gkw714,PMID: 27530427

Lescoute A, Westhof E. 2006. Topology of three-way junctions in folded RNAs. RNA 12:83-93. DOI: https://doi. org/10.1261/rna.2208106, PMID: 16373494

Longtine MS, McKenzie A, Demarini DJ, Shah NG, Wach A, Brachat A, Philippsen P, Pringle JR. 1998. Additional modules for versatile and economical PCR-based gene deletion and modification in Saccharomyces cerevisiae. 
Yeast 14:953-961. DOI: https://doi.org/10.1002/(SICI)1097-0061(199807)14:10<953::AID-YEA293>3.0.CO;2-U, PMID: 9717241

McCaughan UM, Jayachandran U, Shchepachev V, Chen ZA, Rappsilber J, Tollervey D, Cook AG. 2016. Pre-40S ribosome biogenesis factor Tsr1 is an inactive structural mimic of translational GTPases. Nature Communications 7:11789. DOI: https://doi.org/10.1038/ncomms11789, PMID: 27250689

Mohan S, Donohue JP, Noller HF. 2014. Molecular mechanics of 30 S subunit head rotation. PNAS 111:1332513330. DOI: https://doi.org/10.1073/pnas.1413731111, PMID: 25187561

Murshudov GN, Vagin AA, Dodson EJ. 1997. Refinement of macromolecular structures by the maximumlikelihood method. Acta Crystallographica Section D Biological Crystallography 53:240-255. DOI: https://doi. org/10.1107/S0907444996012255, PMID: 15299926

Nicastro G, Taylor IA, Ramos A. 2015. KH-RNA interactions: back in the groove. Current Opinion in Structural Biology 30:63-70. DOl: https://doi.org/10.1016/j.sbi.2015.01.002, PMID: 25625331

Nissan TA, Bassler J, Petfalski E, Tollervey D, Hurt E. 2002. 60S pre-ribosome formation viewed from assembly in the nucleolus until export to the cytoplasm. The EMBO Journal 21:5539-5547. DOI: https://doi.org/10.1093/ emboj/cdf547, PMID: 12374754

Ogle JM, Carter AP, Ramakrishnan V. 2003. Insights into the decoding mechanism from recent ribosome structures. Trends in Biochemical Sciences 28:259-266. DOI: https://doi.org/10.1016/S0968-0004(03)00066-5, PMID: 12765838

Pettersen EF, Goddard TD, Huang CC, Couch GS, Greenblatt DM, Meng EC, Ferrin TE. 2004. UCSF Chimera-a visualization system for exploratory research and analysis. Journal of Computational Chemistry 25:1605-1612. DOI: https://doi.org/10.1002/jcc.20084, PMID: 15264254

Schluenzen F, Tocilj A, Zarivach R, Harms J, Gluehmann M, Janell D, Bashan A, Bartels H, Agmon I, Franceschi F, Yonath A. 2000. Structure of functionally activated small ribosomal subunit at 3.3 angstroms resolution. Cell 102:615-623. DOI: https://doi.org/10.1016/S0092-8674(00)00084-2, PMID: 11007480

Schäfer T, Strauss D, Petfalski E, Tollervey D, Hurt E. 2003. The path from nucleolar $90 \mathrm{~S}$ to cytoplasmic $40 \mathrm{~S}$ preribosomes. The EMBO Journal 22:1370-1380. DOI: https://doi.org/10.1093/emboj/cdg121, PMID: 12628929

Schäfer T, Maco B, Petfalski E, Tollervey D, Böttcher B, Aebi U, Hurt E. 2006. Hrr25-dependent phosphorylation state regulates organization of the pre-40S subunit. Nature 441:651-655. DOI: https://doi.org/10.1038/ nature04840, PMID: 16738661

Strunk BS, Loucks CR, Su M, Vashisth H, Cheng S, Schilling J, Brooks CL, Karbstein K, Skiniotis G. 2011. Ribosome assembly factors prevent premature translation initiation by $40 \mathrm{~S}$ assembly intermediates. Science 333:1449-1453. DOI: https://doi.org/10.1126/science.1208245, PMID: 21835981

Sun Q, Zhu X, Qi J, An W, Lan P, Tan D, Chen R, Wang B, Zheng S, Zhang C, Chen X, Zhang W, Chen J, Dong MQ, Ye K. 2017. Molecular architecture of the $90 \mathrm{~S}$ small subunit pre-ribosome. eLife 6:e22086. DOI: https:// doi.org/10.7554/eLife.22086, PMID: 28244370

Vanrobays E, Gelugne JP, Gleizes PE, Caizergues-Ferrer M. 2003. Late cytoplasmic maturation of the small ribosomal subunit requires RIO proteins in Saccharomyces cerevisiae. Molecular and Cellular Biology 23:20832095. DOI: https://doi.org/10.1128/MCB.23.6.2083-2095.2003, PMID: 12612080

Vanrobays E, Gélugne JP, Caizergues-Ferrer M, Lafontaine DL. 2004. Dim2p, a KH-domain protein required for small ribosomal subunit synthesis. RNA 10:645-656. DOI: https://doi.org/10.1261/rna.5162204, PMID: 15037774

Wimberly BT, Brodersen DE, Clemons WM, Morgan-Warren RJ, Carter AP, Vonrhein C, Hartsch T, Ramakrishnan V. 2000. Structure of the $30 \mathrm{~S}$ ribosomal subunit. Nature 407:327-339. DOI: https://doi.org/10.1038/35030006, PMID: 11014182

Woolford JL, Baserga SJ. 2013. Ribosome biogenesis in the yeast Saccharomyces cerevisiae. Genetics 195:643681. DOI: https://doi.org/10.1534/genetics.113.153197, PMID: 24190922

Woolls HA, Lamanna AC, Karbstein K. 2011. Roles of Dim2 in ribosome assembly. Journal of Biological Chemistry 286:2578-2586. DOI: https://doi.org/10.1074/jbc.M110.191494, PMID: 21075849

Zemp I, Kutay U. 2007. Nuclear export and cytoplasmic maturation of ribosomal subunits. FEBS Letters 581: 2783-2793. DOI: https://doi.org/10.1016/j.febslet.2007.05.013, PMID: 17509569

Zhang K. 2016. Gctf: Real-time CTF determination and correction. Journal of Structural Biology 193:1-12. DOI: https://doi.org/10.1016/j.jsb.2015.11.003, PMID: 26592709

Zheng SQ, Palovcak E, Armache JP, Verba KA, Cheng Y, Agard DA. 2017. MotionCor2: anisotropic correction of beam-induced motion for improved cryo-electron microscopy. Nature Methods 14:331-332. DOI: https://doi. org/10.1038/nmeth.4193, PMID: 28250466 